\title{
Presencia y Biometría de Bazo Accesorio en Individuos Chilenos: Estudio por Tomografía Computarizada
}

\author{
Presence and Biometry of Accessory Spleen in Chile \\ Individuals: Study by Computerized Tomography
}

\author{
Ortega, M. ${ }^{1}$; Alarcón-Muñoz, E. ${ }^{2}$; Ulloa, S. ${ }^{2}$; Cordova, J. ${ }^{2}$; Vidal, N. ${ }^{3}$ \& Olave, E. ${ }^{4}$
}

ORTEGA, M.; ALARCÓN-MUÑOZ, E.; ULLOA, S.; CORDOVA, J.; VIDAL, N. \& OLAVE, E. Presencia y biometría de bazo accesorio en individuos chilenos: estudio por tomografía computarizada. Int. J. morphol., 38(3):787-792, 2020.

RESUMEN: El bazo se localiza en el cuadrante superior izquierdo del abdomen, relacionándose posteriormente con la 9a a 11a costilla, de las que se separa por el diafragma y el receso costodiafragmático, se localiza por detrás del estómago y lateralmente al riñón izquierdo. Por alteraciones en su desarrollo pueden generarse bazos accesorios (BA), considerándose un tejido ectópico del bazo. Se consideran tejido normal, con los mismos procesos fisiológicos que el bazo principal. Con el propósito de localizar y determinar aspectos biométricos de los mismos, se realizó un estudio de corte transversal y de carácter descriptivo, sobre una muestra de 220 exámenes de TC pertenecientes a pacientes mayores de 18 años del Hospital Regional Hernán Henríquez Aravena, Temuco, Chile. Para este estudio se excluyeron toda aquellas TC con antecedentes de esplenectomía y lesiones de Bazo o peri-esplénicas. El análisis de los datos mostró una prevalencia de 32,3 \% de BA, pudiendo ser de una única presencia, dos e incluso tres BA por paciente.De un total de 71 personas que tienen al menos un BA, $34(47,89 \%)$ fueron de sexo femenino y 37 (52,11\%) de sexo masculino. Hubo 56 pacientes (78,9 \%) con un BA, 29 $(40,85 \%)$ del sexo femenino y $27(38,03 \%)$ del masculino; $15(21,1 \%)$ presentaron más de un BA, $5(7,04 \%)$ de sexo femenino y $10(14,08 \%)$ de sexo masculino, si bien se puede observar variación en la cantidad de BA según sexo, no existe una relación estadísticamente significativa entre dichas variables. La ubicación más frecuente encontrada en el plano axial fue la zona antero-medial con 59 casos (66,29 \%); asimismo, en el plano sagital, la localización más frecuente fue en el polo inferior con 40 casos $(44,44 \%)$. Datos biométricos de estos BA son mostrados en Tablas. Esta información será de gran valor morfológico y médico debido a la escasa literatura existente sobre esta materia en individuos chilenos.

PALABRAS CLAVE: Anatomía, Bazo Accesorio, Imagenología, Biometría.

\section{INTRODUCCION}

El bazo es un órgano linfático que contiene una gran cantidad de sangre que se expulsa constantemente a la circulación; en el adulto suele medir $12 \mathrm{~cm}$ de largo, $7 \mathrm{~cm}$ de ancho, 3-4 cm de grosor y posee un peso promedio de 80300 g. El bazo adopta una posición intraperitoneal en el cuadrante superior izquierdo del abdomen, se relaciona posteriormente con la $9^{\mathrm{a}}$ a $11^{\mathrm{a}}$ costilla, de las que se separa por el diafragma y el receso costodiafragmático; por anterior se relaciona con el estómago; por inferior con el ángulo cólico izquierdo, por su cara medial con el riñón izquierdo y por el hilio esplénico con la cola del páncreas (Standring, 2008; Moore et al., 2013).
El bazo comienza su crecimiento en la quinta semana de la vida fetal, a partir de una masa de células mesenquimatosas originada en el mesogástrio dorsal, siendo las alteraciones en su desarrollo las responsables de la aparición de hendiduras, lobulaciones y la generación de bazos accesorios (BA). Estos últimos se consideran un tejido ectópico del bazo, con su propio sistema arterial y venoso, que se produce por la imperfecta fusión de las masas que van a formar el bazo principal en el embrión. El BA se considera un tejido normal, y que actúa bajo los mismos procesos fisiológicos que el bazo principal (Yildiz et al., 2013; Castillo \& Pizzi, 2013).

\footnotetext{
${ }^{1}$ Tecnólogo Médico, con mención en Imagenología.

2 Tecnología Médica, Universidad de La Frontera, Temuco, Chile.

${ }^{3}$ Escuela de Enfermería, Facultad de Salud, Universidad Santo Tomás, Chile.

${ }^{4}$ Facultad de Medicina, Universidad de La Frontera, Temuco, Chile.
} 
El diagnóstico habitual de BA es principalmente por hallazgo incidental en las diferentes ramas de la imagenología médica, siendo la técnica imagenológica de tomografía computarizada (TC) la más utilizada, la cual utiliza radiación ionizante que permite la identificación de órganos internos en los tres planos. La TC puede definirse como un examen radiológico capaz de reproducir imágenes tomográficas finas que representan reconstrucciones matemáticas computarizadas de los tejidos y órganos del cuerpo (Bontrager \& Lampignano, 2014). Además, este examen permite hacer un diagnóstico diferencial de BA, observándose como una masa con márgenes bien definidos, forma homogénea y de igual densidad que el bazo principal (Castillo \& Pizzi).

La prevalencia general del BA mediante estudios imagenológicos es de 16,6\% y mediante exploración intraoperatoria de un 14,6 \%, porcentaje que varía según la ubicación geográfica. Oceanía posee una prevalencia de 26,6 $\%$, Norteamérica de $16,7 \%$, y Asia de 14,1\%. En nuestro país no existe un estudio que declare la prevalencia de BA en población chilena (Vikse et al., 2017).

La detección y caracterización de los BA es importante debido a tres razones: 1) Los BA pueden ser sintomáticos, presentando torsión, ruptura espontánea, hemorragia o formación de quistes. 2) Su detección es fundamental en pacientes con enfermedades hematológicas que requieren esplenectomía y que podrían quedar sub tratados de no resecarse todo el tejido esplénico funcional y 3) Los bazos accesorios pueden confundirse con adenopatías linfáticas o tumores de otros órganos abdominales (Mortelé et al., 2004; Castillo \& Pizzi).

Es por ello, que el objetivo de este estudio ha sido determinar la prevalencia, cantidad, localización, biometría y atenuación de los BA en individuos chilenos, además de relacionar estas variables con respecto al sexo.

Esta información será de gran valor morfológico y médico debido a la escasa literatura existente sobre esta materia en individuos chilenos.

\section{MATERIAL Y METODO}

Con el fin de cumplir el objetivo de este trabajo, se realizó un estudio de corte transversal y de carácter descriptivo, sobre una muestra de 220 exámenes de TC pertenecientes a pacientes mayores de 18 años del Hospital Regional Hernán Henríquez Aravena. Para este estudio se excluyeron toda aquellas TC con antecedentes de esplenectomía y lesiones de Bazo o peri-esplénicas.
Si bien no existe un protocolo de TC definido específicamente para realizar la identificación y caracterización del BA, se hizo uso del protocolo establecido en el centro donde se hizo el estudio.

El análisis tomográfico se realizó utilizando planos axiales, en fase venosa y sin contraste; y planos coronales en fase venosa. Los espesores de corte de las imágenes axiales fueron de $2 \mathrm{~mm}$ en fase venosa y arterial; mientras que, para los planos axiales sin contraste, el espesor de corte fue de $5 \mathrm{~mm}$. Los planos coronales y sagitales, en todas las fases, fueron de un espesor de corte de $5 \mathrm{~mm}$.

Esta investigación fue autorizada por el Comité de Etica correspondiente.

\section{Criterios utilizados para evaluar la presencia de un BA:}

Características Radiológicas: Se observa como una Masa bien delimitada, con atenuación y/o captación de contraste similar al bazo principal en todas sus fases (Izzo et al., 2004; Kim et al., 2008; Yang et al., 2017), obteniendo el valor de atenuación con y sin contraste (fase venosa) tanto del bazo accesorio como del bazo principal.

Homogeneidad: Característica en donde el BA es visualmente homogéneo en fase venosa (Izzo et al.).

Tamaño: Generalmente estas masas son de tamaño cercano a los $20 \mathrm{~mm}$, las menores a $10 \mathrm{~mm}$ tienden a observarse con menor atenuación que el Bazo principal (Mortelé et al.).

Localización: Para la localización nos basamos en el método descrito por Mortelé et al. en el cual, el plano axial, es dividido en 6 compartimientos anatómicos discretos, ubicados en relación al bazo principal. Se traza una línea perpendicular al eje largo del bazo principal, a nivel del hilio esplénico, generándose los compartimientos antero medial (AM), antero-lateral (AL), postero-medial (PM), y postero-lateral (PL), así como también anterior (A) y posterior $(\mathrm{P})$. En el plano coronal, dividimos el bazo principal en tercios, polo superior (PS: tercio superior, superior al hilio), hilio (H: tercio medio) y polo inferior (PI: tercio inferior, inferior al hilio). En los casos polo superior y polo inferior se incluyó también a los que estaban superior e inferior al bazo principal (más allá de los polos) respectivamente (Fig. 1).

Diámetro: Medimos diámetros antero-posteriores, laterolaterales y supero-inferiores.

Número de BA: Se determinó la cantidad de BA. 
ORTEGA, M.; ALARCón-MUÑOZ, E.; ULLOA, S.; CORDOVA, J.; VIDAL, N. \& OLAVE, E. Presencia y biometria de bazo accesorio en individuos chilenos: estudio por tomografia computarizada. Int. J. morphol., 38(3):787-792, 2020

Análisis estadístico: Los datos obtenidos fueron recopilados digitalmente mediante el programa Microsoft Excel 2016 y analizados en el software estadístico SPSS (IBM SPSS Statistics, v 21).

El análisis descriptivo de las variables se realizó a través de medidas de tendencia central, tablas de frecuencia y de contingencia. El análisis de relación entre las variables cualitativas y sexo se realizó mediante la prueba estadística Chi-cuadrado $(\mathrm{p}<0,05)$. Finalmente, para establecer la existencia de diferencias de medias entre variables cuantitativas y sexo, se realizó la prueba de normalidad Kolmogorov-Smirnov para determinar la existencia o no de normalidad, de existir normalidad se aplicó la prueba estadística de $\mathrm{t}$ de student $(\mathrm{p}<0,05)$, de lo contrario se utilizó la prueba estadística de U de Mann-Whitney.
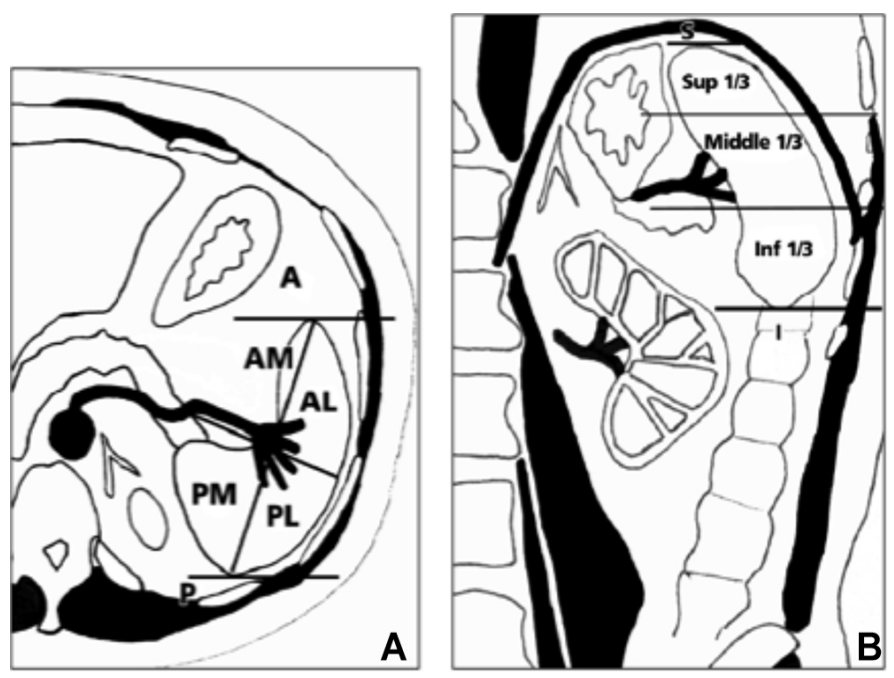

Fig. 1. Compartimientos anatómicos definidos en axial (A) y sagital (B).

\section{RESULTADOS}

Prevalencia de bazos accesorios. El análisis de los datos obtenidos mostró una prevalencia de 32,3\% de bazos accesorios en la población bajo estudio, pudiendo ser de una única presencia, dos e incluso tres BA por paciente (Figs. 2 y 3). De un total de 71 personas que poseían al menos un BA, $34(47,89 \%)$ de ellas corresponde al sexo femenino y $37(52,11 \%)$ al sexo masculino, si bien existe una diferencia en la presencia según sexo esta no es estadísticamente significativa $\mathrm{p}=0,98$ (Tabla VI).

La presencia de BA mostró que 56 pacientes $(78,9 \%)$ presentaban un BA, $29(40,85 \%)$ del sexo femenino y $27(38,03 \%)$ del sexo masculino y 15 pacientes $(21,1 \%)$ más de un BA, $5(7,04 \%)$ de sexo femenino y $10(14,08 \%)$ de sexo masculino. De estos pacientes, 12 de ellos tenían 2 BA y los 3 restantes tenían 3 BA. Si bien se puede observar variación en la cantidad de BA según sexo, no existe una relación estadísticamente significativa entre dichas variables $\mathrm{p}=$ 0,20 (Tabla VI).

Dimensiones de bazos accesorios. Las dimensiones de los 89 BA fueron tomadas en los ejes antero-posterior, latero-lateral y supero-inferior de toda la población bajo estudio y por sexo, datos expresados en las Tablas I, II y III. El volumen promedio que ocupan los BA es de 345,61 mm3. siendo mayor en el sexo femenino con 387,35 mm3. que en el sexo masculino 309,95 mm3, sin existir diferencia estadística entre ellos p>0,05 (Tabla VI).
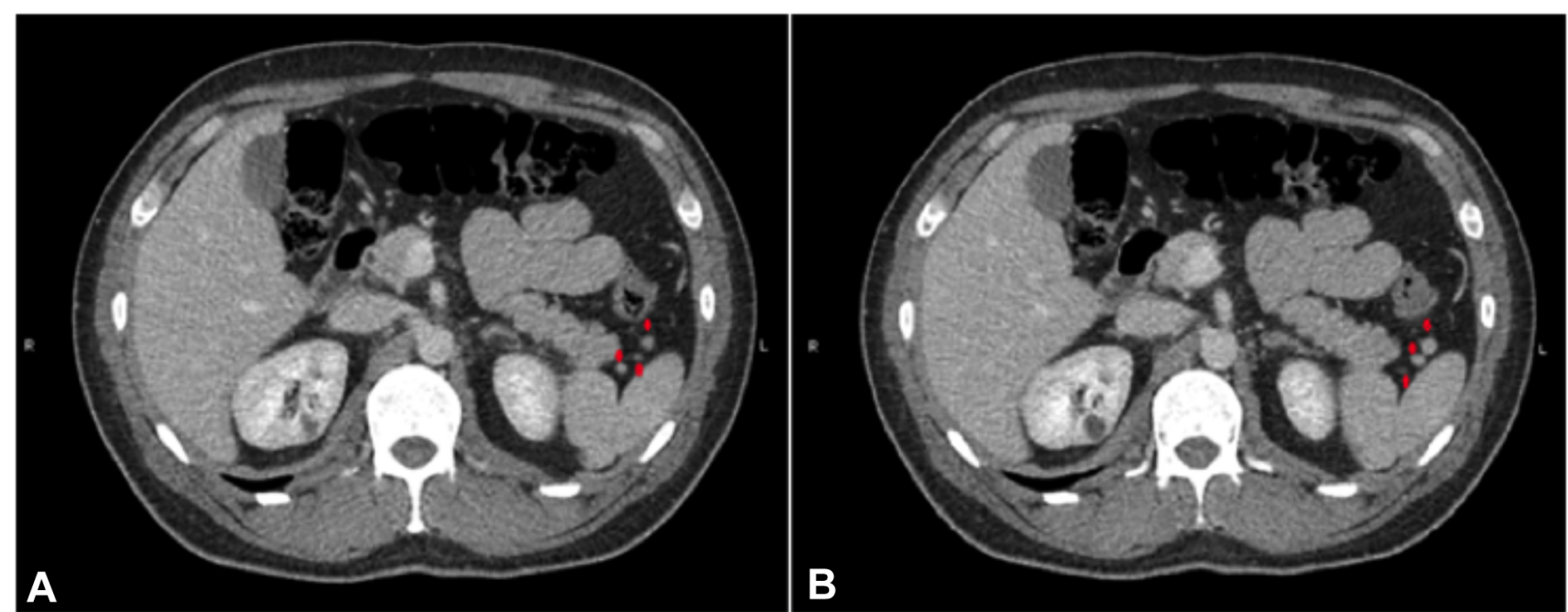

Fig. 2. A y B: Mismo individuo con tres BA en localización antero medial. (Flechas rojas) 

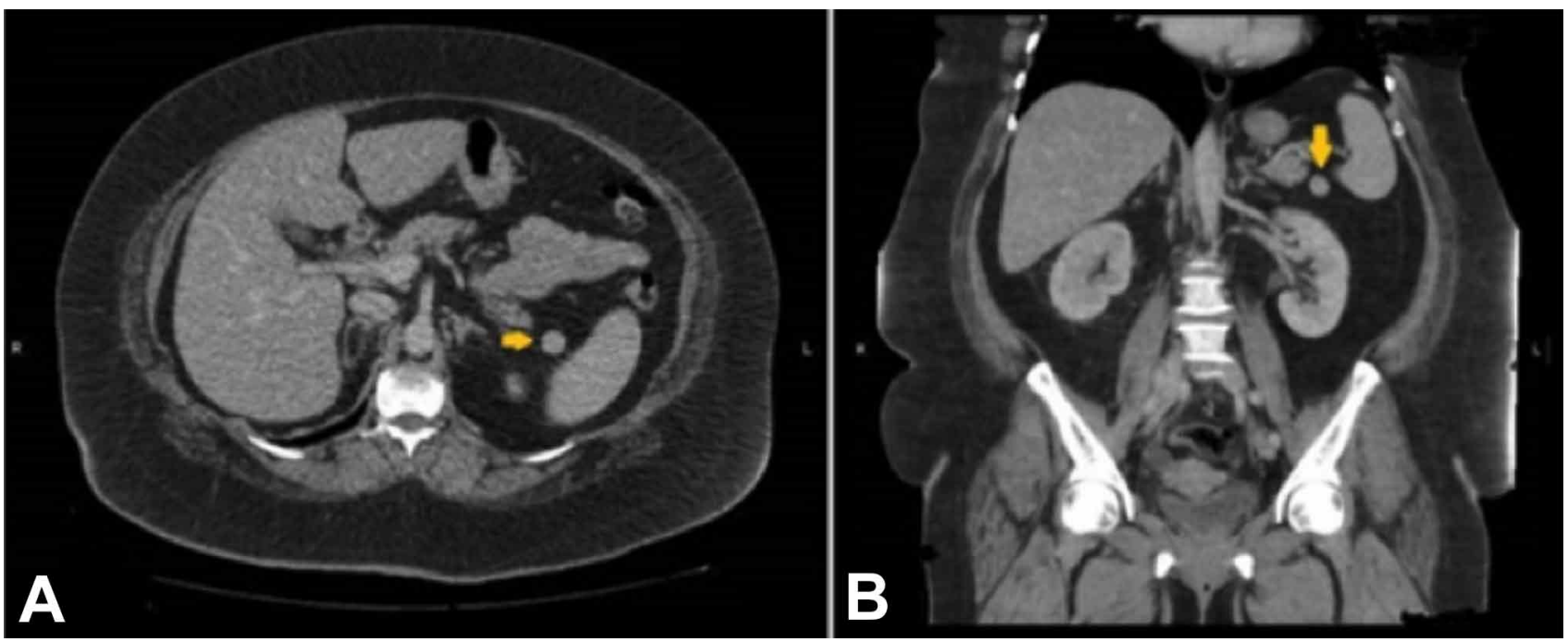

Fig. 3. BA único en corte axial(A) y coronal(B). (Flechas Amarillas.

Tabla I. Dimensiones y volumen de BA (mm).

\begin{tabular}{ccccc}
\hline Medida & Mínimo & Máximo & Media & Desviación estándar \\
\hline Antero Posterior & 2,42 & 15,91 & 7,67 & 3,21 \\
Latero Lateral & 2,92 & 16,46 & 7,99 & 3,07 \\
Supero Inferior & 2,65 & 14,98 & 7,61 & 2,89 \\
Volumen $\left(\mathrm{mm}^{3}\right)$ & 9,89 & 2058,65 & 345,61 & 402,82 \\
\hline
\end{tabular}

Tabla II. Dimensiones y volumen de BA en pacientes femeninos (mm).

\begin{tabular}{ccccc}
\hline Medida & Mínimo & Máximo & Media & Desviación estándar \\
\hline Antero Posterior & 3,59 & 15,50 & 8,02 & 3,48 \\
Late ro Lateral & 3,88 & 15,29 & 8,41 & 3,19 \\
Supero Inferior & 3,91 & 14,24 & 8,07 & 3,20 \\
Volumen $\left(\mathrm{mm}^{3}\right)$ & 29,54 & 1540,72 & 387,35 & 424,67 \\
\hline
\end{tabular}

Tabla III. Dimensiones y volumen de BA en pacientes masculinos (mm).

\begin{tabular}{ccccc}
\hline \multicolumn{1}{c}{ Medida } & Mínimo & Máximo & Media & Des viación estándar \\
\hline Antero Posterior & 2,42 & 15,91 & 7,36 & 2,96 \\
Latero Lateral & 2,92 & 16,46 & 7,63 & 2,95 \\
Supero Inferior & 2,65 & 14,98 & 7,22 & 2,56 \\
Volumen $\left(\mathrm{mm}^{3}\right)$ & 9,89 & 2058,65 & 309,95 & 384,07 \\
\hline
\end{tabular}

Tabla IV. Ubicación de bazos accesorios en los planos axial y coronal.

\begin{tabular}{|c|c|c|c|c|c|c|c|}
\hline$x^{2}$ & Anterior & $\begin{array}{l}\text { Antero } \\
\text { lateral }\end{array}$ & $\begin{array}{l}\text { Antero } \\
\text { medial }\end{array}$ & $\begin{array}{l}\text { Postero } \\
\text { lateral }\end{array}$ & $\begin{array}{l}\text { Postero } \\
\text { medial }\end{array}$ & Posterior & Total \\
\hline Coronal & & & & & & & \\
\hline Polo superior & 1 & 3 & 15 & 0 & 0 & 0 & 19 \\
\hline Polo medio & 2 & 8 & 17 & 0 & 2 & 1 & 30 \\
\hline Polo inferior & 2 & 7 & 27 & 2 & 2 & 0 & 40 \\
\hline Total & 5 & 18 & 59 & 2 & 4 & 1 & 89 \\
\hline
\end{tabular}


ORTEGA, M.; ALARCÓN-MUÑOZ, E.; ULLOA, S.; CORDOVA, J.; VIDAL, N. \& OLAVE, E. Presencia y biometria de bazo accesorio en individuos chilenos: estudio por tomografia computarizada. Int. J. morphol., 38(3):787-792, 2020.

Tabla V. Atenuación (UH) de BA y bazo principal.

\begin{tabular}{lccccc}
\hline Fase de lectura de atenuación & N & $\begin{array}{c}\text { Mínima } \\
(\mathrm{UH})\end{array}$ & $\begin{array}{c}\text { Máxima } \\
(\mathrm{UH})\end{array}$ & $\begin{array}{c}\text { Media } \\
(\mathrm{UH})\end{array}$ & $\begin{array}{c}\text { Desviación } \\
\text { estándar }\end{array}$ \\
\hline Bazo Accesorio C/Contraste & 89 & 20,80 & 126,25 & 88,79 & 20,72 \\
Bazo Principal C/Contraste & 89 & 10,00 & 139,15 & 105,18 & 15,77 \\
Bazo Accesorio S/Contraste & 89 & 5,33 & 60,64 & 32,80 & 14,66 \\
Bazo Principal S/Contraste & 89 & 40,60 & 67,04 & 52,66 & 5,33 \\
\hline
\end{tabular}

Tabla VI. Pruebas estadísticas.

\begin{tabular}{lccc}
\hline Variable & Chi-Cuadrado & $\begin{array}{c}\text { Prueba de } \\
\text { Normalidad (KS) }\end{array}$ & $\begin{array}{c}\text { U de Mann- } \\
\text { Whitney }\end{array}$ \\
\hline Presencia según sexo & $\mathrm{p}=0,98$ & - & - \\
Cantidad según sexo & $\mathrm{p}=0,20$ & - & - \\
Localización coronal según sexo & $\mathrm{P}=0,385$ & - & - \\
Localización axial según sexo & $\mathrm{P}=0,063$ & - & - \\
Volumen de los bazo accesorios según sexo & - & $\mathrm{P}=0,000$ & $\mathrm{P}<0,05$ \\
\hline
\end{tabular}

\section{DISCUSIÓN}

El BA es una masa generalmente ovalada y considerada un tejido ectópico del bazo, que se forma en la quinta semana de la vida fetal al ocurrir una imperfección en la unión de las masas que formarán el bazo en el feto. Su presencia puede gatillar problemas en el trascurso de la vida del paciente, por lo que es de vital importancia su detección y conocimiento de su prevalencia en la población chilena (Mortelé et al.; Castillo \& Pizzi).

Nuestro estudio detectó una prevalencia de 32,4\% de al menos un BA en pacientes de población chilena, sin diferencias significativas según sexo. Este resultado es superior a la reportada por Mortelé et al., que de un total de 1000 pacientes estudiados 156 pacientes tenían a lo menos $1 \mathrm{BA}$, es decir, una prevalencia del 15,6\%, sin embargo, su metodología fue diferente ya que utilizaron un grosor de corte de 8 mm. Romer \& Wiesner (2012), encontraron a lo menos 1 BA en 199 pacientes de 1735 que fueron estudiados, con una prevalencia de $11,5 \%$, con un grosor de corte de $5 \mathrm{~mm}$, al igual que en nuestro estudio. La mayor prevalencia encontrada en nuestra población puede deberse a que utilizamos un grosor de corte menor. La comparación con otros autores puede ser revisada en la Tabla VII.

El diámetro del BA descrito por Mortelé et al., muestra una variación de 4 a $29 \mathrm{~mm}$, con un promedio de 11,9 mm de diámetro en el sentido antero-posterior, mientras que en el sentido latero-lateral varía de 4 a $25 \mathrm{~mm}$, con un promedio de $11,6 \mathrm{~mm}$; por otra parte, el diámetro cráneo-caudal varía de 8 a $32 \mathrm{~mm}$, con un promedio de $16,8 \mathrm{~mm}$.

Según Rashid (2014)el diámetro promedio de un bazo ectópico corresponde a 14,7 mm, variando de 3 a $79 \mathrm{~mm}$.

En nuestro estudio, el promedio de los diámetros antero-posterior varió de 2,42 $\mathrm{mm}$ a $15,91 \mathrm{~mm}$ con un promedio de 7,67 mm, mientras que el promedio de diámetro laterolateral varió de 2,92 a 16,46 mm con un promedio de 7,99 $\mathrm{mm}$; por último, el promedio de los diámetros supero-inferior varió de 2,65 a 14,98 mm con un promedio de 7,61 mm. Los rangos y promedio de diámetro fueron menores en nuestro estudio, esto también se puede explicar por grosor de corte utilizado, que en nuestro caso fue de $2 \mathrm{~mm}$. Cuando mayor es el grosor de corte, se enmascaran BA de mayor tamaño, disminuyendo la sensibilidad en la detección de éstos, ya que es mayor el volumen parcial generado en las imágenes, dificultando la determinación visual del límite de los BA encontrados, esto genera que los BA de menor tamaño (esto es, menor a $2 \mathrm{~mm}$ ) no sean identificables.

En la literatura consultada, no existen trabajos que se refieran al volumen de los BA, variable que si fue abordada en nuestro estudio. Como resultado obtuvimos un promedio de $345,61 \mathrm{~mm}^{3}$, con una variación entre $9,89 \mathrm{~mm}^{3}$ y 2058,65 $\mathrm{mm}^{3}$.

Tabla VII. Comparación de prevalencia entre autores.

\begin{tabular}{lccccc}
\hline Autores & Año & Técnica & $\begin{array}{c}\text { Pacientes } \\
\text { totales }\end{array}$ & $\begin{array}{c}\text { Pacientes con } \\
\text { BA detectado }\end{array}$ & Prevalencia (\%) \\
\hline Mortelé et al. & 2004 & TC & 1000 & 156 & 15,6 \\
Quah et al. & 2011 & TC & 58 & 11 & 19 \\
Romer \& Wiesner. & 2012 & TC & 1735 & 199 & 11,5 \\
Abdulrahman. & 2014 & TC & 334 & 63 & 18,8 \\
\hline
\end{tabular}


La ubicación más común del BA según Mortelé et al. es en el tercio inferior del compartimiento postero-medial del bazo, a diferencia de nuestro estudio, donde se determinó que la ubicación más común en la población chilena, en el plano axial, fue el eje antero-medial con 59 casos $(66,29 \%)$; asimismo, en el plano sagital, la localización más frecuente fue en el polo inferior con 40 casos $(44,44 \%)$. La intersección entre los dos planos con más presencia de BA fue entre la zona antero medial y el polo inferior con 27 casos $(33,34 \%)$.

Con respecto a las Unidades Hounsfield determinadas en este estudio, podemos decir que la media de los BA encontrados en fase venosa (con contraste) fue de 88,79 $\pm 20,72$ UH y sin contraste fue de $32,80 \pm 14,66 \mathrm{UH}$, mientras en el bazo principal presentó en la fase venosa una media de 105,18 $\pm 15,77 \mathrm{UH}$ y sin contraste de 52,66 \pm 5,33 UH. Estos valores no fueron encontrados en la literatura consultada para ser contrastados. Basados en estos datos podemos decir cuándo es o no es un BA, considerando que podemos compararlo con el bazo principal y no haber mayor variación en su captación, y así diferenciarlo de otros tejidos sanos o tumorales. La importancia de esta relación recae en que, sin necesidad de realizar intervenciones invasivas al paciente, se puede determinar de que tejido y órgano se trata.

Como conclusión, el presente estudio nos permite determinar que el bazo accesorio debería ser un hallazgo incidental considerable, por su alta prevalencia en la población chilena y su relevancia en pacientes con patologías inmunológicas o tumorales que puedan confundirse con éste, por su forma y/o tamaño. Por otro lado, la ubicación más común en la población en estudio es en el polo inferior y anteromedial del bazo principal, ubicación que debe considerarse al momento de alguna cirugía o para determinar algún diagnóstico diferencial con alguna masa, lo cual mezclado con los datos de UH, ayudaría a evitar posibles intervenciones invasivas por diagnósticos erróneos de patologías o tumores.

ORTEGA, M.; ALARCÓN-MUÑOZ, E.; ULLOA, S.; CORDOVA, J.; VIDAL, N. \& OLAVE, E. Presence and biometry of accessory spleen in chilean individuals: study by computerized tomography. Int. J. Morphol., 38(3):787-792, 2020.

SUMMARY: The spleen is located in the upper left quadrant of the abdomen, subsequently related to the 9 th to 11 th rib, from which it is separated by the diaphragm and the cost-diaphragmatic recess, it is located behind the stomach and laterally to the left kidney. Due to alterations in its development, accessory spleens (AS) can be generated, being considered an ectopic tissue of the spleen. The AS are considered normal tissue, with the same physiological processes as the main spleen. With the purpose of locating and determining biometric aspects of them, a cross-sectional and descriptive study was carried out on a sample of $220 \mathrm{CT}$ scans belonging to patients over 18 years of age at the Hernán Henríquez Aravena Regional Hospital, Temuco, Chile . For this study, all CT scans with a history of splenectomy and spleen or peri-splenic lesions were excluded. The analysis of the data showed a prevalence of $32.3 \%$ of AS, being able to be of a single presence, two and even three AS per patient. Of a total of 71 people who have at least one AS, $34(47.89 \%)$ were female and 37 (52.11 \%) male. There were 56 patients $(78.9 \%)$ with a one AS, $29(40.85 \%)$ of the female sex and 27 $(38.03 \%)$ of the male; $15(21.1 \%)$ presented more than one AS, $5(7.04 \%)$ female and $10(14.08 \%)$ male, although variation in the amount of AS according to sex can be observed, no there is a statistically significant relationship between these variables. The most frequent location found in the axial plane was the anteromedial zone with 59 cases (66.29\%); also, in the sagittal plane, the most frequent location was in the lower pole with 40 cases $(44.44 \%)$. Biometric data of these $\mathrm{AS}$ are shown in tables. This information will be of great morphological and medical value due to the limited existing literature on this subject in Chilean individuals.

KEY WORDS: Anatomy; Accessory Spleen; Imaging; Biometry.

\section{REFERENCIAS BIBLIOGRÁFICAS}

Abdulrahman, S. Accessory spleen: prevalence and multidetector CT appearance. Malays. J. Med. Sci., 21(4):18-23, 2014.

Bontrager, K. \& Lampignano, J. Textbook of Radiographic Positioning and Related Anatomy. 5th ed. St. Louis (Mo.), Elsevier/Mosby, 2014

Castillo, C. O. A. \& Pizzi, L. P. Bazo accesorio simulando tumor suprarrenal. Rev. Chil. Cirug., 65(2):162-5, 2013.

Izzo, L.; Caputo, M. \& Galati, G. Intrahepatic accessory spleen: imaging features. Liver Int., 24(3):216-7, 2004.

Kim, S. H.; Lee, J. M.; Han, J. K.; Lee, J. Y.; Kim, K. W.; Cho, K. C. \& Choi, B. I. Intrapancreatic accessory spleen: findings on MR Imaging, CT, US and scintigraphy, and the pathologic analysis. Korean J. Radiol., 9(2):162-74, 2008.

Moore, K. L.; Dalley, A. F. \& Agur, A. M. R. Anatomía con Orientación Clínica. 7th ed. Barcelona, Wolters Kluwer, Lippincott Williams \& Wilkins, 2013.

Mortelé, K. J.; Mortelé, B. \& Silverman, S. G. CT features of the accessory spleen. A. J. R. Am. J. Roentgenol., 183(6):1653-7, 2004.

Quah, C.; Ayiomamitis, G.; Shah, A. \& Ammori, B. Computed tomography to detect accessory spleens before laparoscopic splenectomy: is it necessary? Surg. Endoscopic, 25:261-5, 2011.

Rashid, S. Accessory spleen: Prevalence and multidetector CT appearance. Malay J. Med. Scie., 21:18-23, 2014.

Romer, T. \& Wiesner, W. The accessory spleen: prevalence and imaging findings in 1,735 consecutive patients examined by multidetector computed tomography. JBR-BTR, 95(2):61-5, 2012.

Standring, S. Gray's Anatomy. 40th ed. Edinburgh, Churchill Livingstone/ Elsevier, 2008

Vikse, J.; Sanna, B.; Henry, B. M.; Taterra, D.; Sanna, S.; Pe kala, P. A.; Walocha, J. A. \& Tomaszewski, K. A. The prevalence and morphometry of an accessory spleen: A meta-analysis and systematic review of 22,487 patients. Int. J. Surg., 45:18-28, 2017.

Yang, B.; Valluru, B.; Guo, Y. R.; Cui, C.; Zhang, P. \& Duan, W. Significance of imaging findings in the diagnosis of heterotopic spleen-an intrapancreatic accessory spleen (IPAS): Case report. Medicine (Baltimore), 96(52):e9040, 2017.

Yildiz, A. E.; Ariyurek, M. O. \& Karcaaltincaba, M. Splenic anomalies of shape, size, and location: pictorial essay. Scientific World Journal, 2013:321810, 2013.

\section{Dirección para Correspondencia}

Prof. Dr. Enrique Olave

Facultad de Medicina

Universidad de La Frontera

Temuco - CHILE

Email: enrique.olave@ufrontera.cl

Recibido: $17-10-2019$

Aceptado:08-01-2020 